This item was submitted to Loughborough's Research Repository by the author.

Items in Figshare are protected by copyright, with all rights reserved, unless otherwise indicated.

\title{
Advantages in using LEDS as the main light source in solar simulators for measuring PV device characteristics
}

PLEASE CITE THE PUBLISHED VERSION

http://dx.doi.org/10.1117/12.795428

PUBLISHER

(c) SPIE

VERSION

AM (Accepted Manuscript)

\section{PUBLISHER STATEMENT}

This work is made available according to the conditions of the Creative Commons Attribution-NonCommercialNoDerivatives 4.0 International (CC BY-NC-ND 4.0) licence. Full details of this licence are available at: https://creativecommons.org/licenses/by-nc-nd/4.0/

\section{LICENCE}

CC BY-NC-ND 4.0

\section{REPOSITORY RECORD}

Bliss, Martin, Thomas R. Betts, and Ralph Gottschalg. 2019. "Advantages in Using LEDS as the Main Light Source in Solar Simulators for Measuring PV Device Characteristics". figshare. https://hdl.handle.net/2134/17777. 


\title{
ADVANTAGES IN USING LEDS AS THE MAIN LIGHT SOURCE IN SOLAR SIMULATORS FOR MEASURING PV DEVICE CHARACTERISTICS
}

\author{
M.Bliss*, T.R. Betts, R. Gottschalg \\ Centre for Renewable Energy Systems Technology (CREST), Department of Electronic and \\ Electrical Engineering, Loughborough University, Loughborough, Leicestershire, LE11 3TU, UK
}

\begin{abstract}
Advances in photovoltaic technology resulted in increased complexity of device calibration, largely being affected by deviations of test spectrum from natural spectra. While the output spectrum of some solar simulators is adjustable, generally only light intensity and module temperature can be varied. This is due to the light sources used in current simulators. LEDs offer an additional degree of freedom, when using an appropriate combination of wavelengths. This paper presents the advantages of this lighting technology for solar simulation and backs these up through results of the prototype unit developed at the Centre for Renewable Energy Systems Technology.

The ability to keep LEDs stable for a long time and dim them with minimal changes in the spectrum allows generation of a spectrum closely matched to AM1.5G standard test spectrum or indeed even realistic variations of the outdoor spectrum. LEDs can be controlled very fast within microseconds or operated continuously, combining a steady state and a flash solar simulator with additional functions such as variable flash frequencies and flash shape. Combined with the life expectancy exceeding 50.000h, LEDs are a strong candidate for solar simulator light sources introducing a significant improvement in calibration lifetime as well as significantly reduced running cost.

The usage of LEDs can enhance today's characteristic measurement functions and even opens possibilities to fully characterise solar cells indoors within a much shorter time than is possible today, over a range of conditions previously only available through outdoor characterisation.
\end{abstract}

Keywords: Light emitting diode, LED, solar simulator, calibration, classification, measurement

\section{INTRODUCTION}

Fast developing research in solar cells and modules leads to growing complexity in device structures which also introduces new challenges in supposedly basic current-voltage characterisation of devices. Two examples are the problems of measuring devices with high cell capacitances ${ }^{[1]}$ such as high efficiency silicon devices or the inability of single source simulators to calibrate multi-junction devices. Steady state solar simulators can overcome the first issue but this comes at a very significant increase of operation cost, and a 'slow flash' solar simulator would be much more desirable to simplify thermal management of the devices under test. These solar simulators also incur high operation and maintenance costs and relatively long down times for recalibration, because the light sources used only have a lifetime of up to $2000 \mathrm{~h}$ and the homogeneity often needs to be re-adjusted for a new set of lamps. The costs are much lower for flash solar simulators. Maintenance costs and down times are largely reduced due to the flash bulb lifetime of around 100.000 pulses. Additionally, problems of thermal instability of the test device are reduced to a minimum, due to the light exposure time in the range of milliseconds, which is too short for any significant device heat up. Also, device preconditioning is virtually impossible with pulse solar simulators.

Generally, devices are tested in laboratories under standard test conditions (STC) with a defined light intensity of $1000 \mathrm{~W} / \mathrm{m}^{2}$, air mass (AM) $1.5 \mathrm{G}$ (global) light spectrum and a device temperature of $25^{\circ} \mathrm{C}^{[2]}$. However, real conditions are different and varying, resulting in operating efficiencies outdoors which typically are lower than the STC efficiency. Today, there is no solar simulator being able to represent realistic spectral changes during operation. While the output

* Tel.: +44 1509 635327, Fax: +44 1509 635301, Email: M.Bliss@lboro.ac.uk 
spectrum of some solar simulators is slightly adjustable, generally only light intensity and module temperature can be varied and the difference between different air-mass spectra or sunny-cloudy differences are not representable. This means that today's solar simulators do not provide the variability of environmental conditions to carry out a robust energy rating of today's devices. In addition, characterisation of multijunction solar devices without introducing large uncertainties is only possible with very few solar simulators which are able to vary the output spectrum, because the average photon energy needs to be adjusted to current match all stacked, series connected cells ${ }^{[3]}$.

All the advantages and disadvantages in today's solar simulators very largely depend on the light sources used to illuminate the test device. A solar simulator with an ideal light source should be able to measure characteristics in steady state and also in pulsed light, providing variable flash times to be able to compensate module heat-up with capacitance effects. A much longer lifetime of the light sources in the simulator is also desirable. A much higher variability of the output spectrum would additionally enable measurements in more realistic outdoor conditions as well as provide better abilities to measure multijunction solar cells. All this and even more can be achieved by replacing conventional light sources with light emitting diodes (LEDs). An LED-based solar simulator prototype, designed and constructed in-house at CREST has shown that the idea of using LEDs is far from trivial, but is possible and could well lead to a new generation of solar simulators providing highly accurate I-V characteristic measurements for all types of devices.

\section{WHY USE LEDS AND WHAT COULD BE ACHIEVED?}

An LED is semiconductor diode that emits a narrow-spectrum light when electrically biased in the forward direction of the p-n junction. This effect is a form of electroluminescence. In the mid 1920s, Olec Losev studied emissions from rectifier diodes used in radio receivers when current was passing through and first discovered the luminescence effect on zinc oxide and silicon carbide diodes. The first LED semiconductor lasers were revealed in 1962 simultaneously from four research groups in the USA ${ }^{[4]}$. Since then, LEDs have become a key component of today's technologies, being used in hundreds of applications such as in CD- and DVD-ROMs, as indicators in virtually all types of equipment, in new traffic and car lights, as large size displays and for transmitting data in fibre optics and TV remotes.

Due to their unique characteristics, LEDs have many advantages over conventional light sources used in today's solar simulators. They also have characteristics which can lead to problems and uncertainties when left unattended and used in a solar simulator. However, these are engineering issues and can be overcome with by using good design and appropriate electronics.

\subsection{Advantages}

First of all LEDs react and can be regulated accurately in matters of microseconds (see Fig. 1 for the measured light control speed in the LED-based solar simulator). They also can be kept stable at one light output intensity continuously for very long time as long as appropriate control and regulation electronics are employed. Thus, a flash solar simulator can be combined with a steady state solar simulator and additional functions such as variable flash frequencies and flash shape can be implemented. The capacitive effects in PV device measurements seen on flash simulators can be prevented without having to suffer temperature instability influences of a steady state simulator. As measured with the LED-based solar simulator prototype and shown in Fig. 2, this also opens possibilities to measure these effects exactly and investigate them through variable and adjustable flash speed and shape. The high stability of LEDs would also overcome instabilities in conventional lamps, such as arc-jumping of xenon arc lamps ${ }^{[5]}$ and are thus a very attractive solution for increasing measurement accuracy.

LEDs have a relatively narrow monochromic output spectrum (except for white LEDs) and are available in a wide variety of colours and wavelengths from ultraviolet to infrared, Unfortunately, anything outside the visual range is seen as a specialist item and thus is very costly at this time. An individual LED cannot meet the spectral standard for solar simulation $^{[2]}$, but combining a number of different colours opens the possibility of creating a closely-matched AM1.5G spectrum as prescribed for calibration of photovoltaic devices. Such a combination also provides the ideal base for high variability of the simulator output spectrum, due to the ability to dim LEDs. By adjusting the intensity of each colour, PV devices can be measured under more realistic conditions in the laboratory, such as under red-rich spectra during sun set or even under diffuse, blue-rich conditions rather than only under AM1.5G. Also, a variable spectrum enables accurate characterisation of multi-junction photovoltaic device. The incident spectrum could be changed easily to a slightly redrich or blue-rich spectra so that all junctions get current matched. At this point the fill factor is at a minimum and the current and maximum power output is at a maximum ${ }^{[6]}$. 


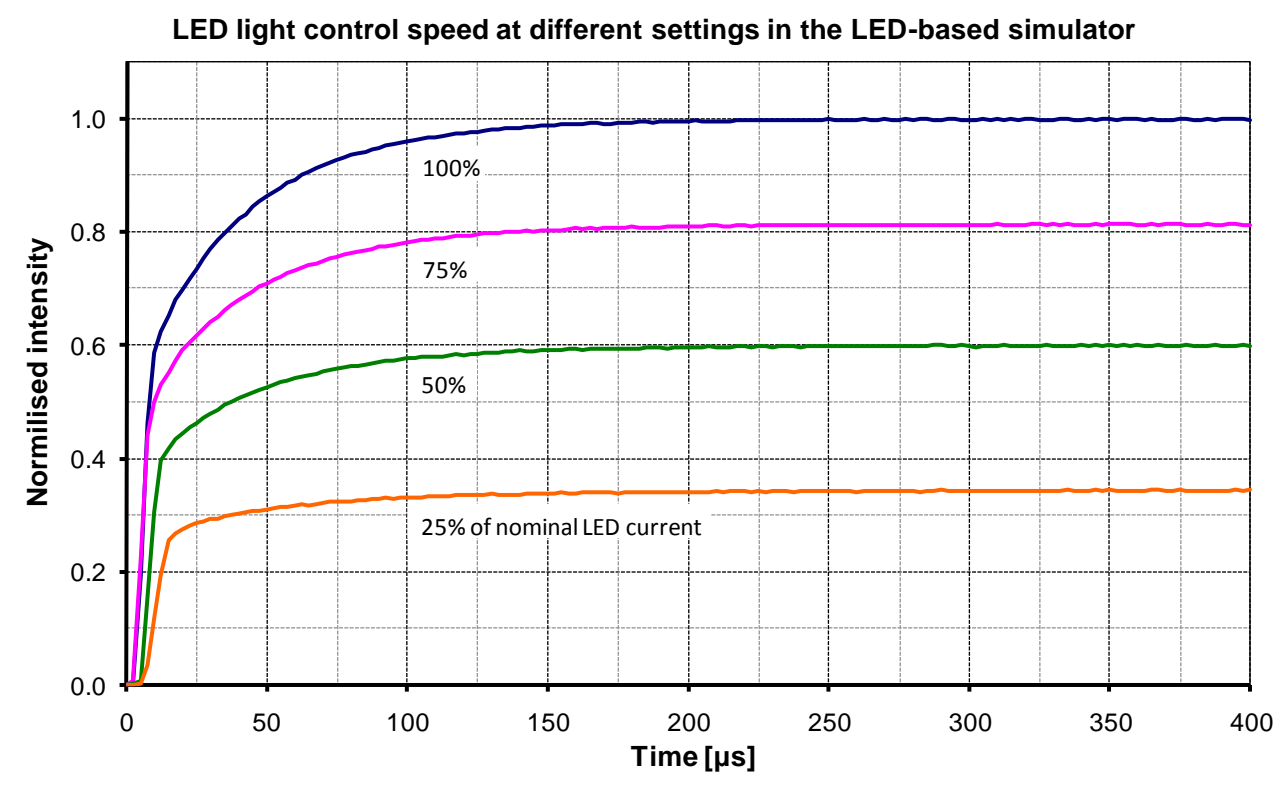

Fig. 1: LED light control speed at different settings of the LED's nominal current; The LED-based solar simulator needs around $220 \mu \mathrm{s}$ to regulate the LEDs current to any required value. The electronics used very largely limit the control speed to achieve highly stable regulation. The LEDs itself can react in less than 100ns.

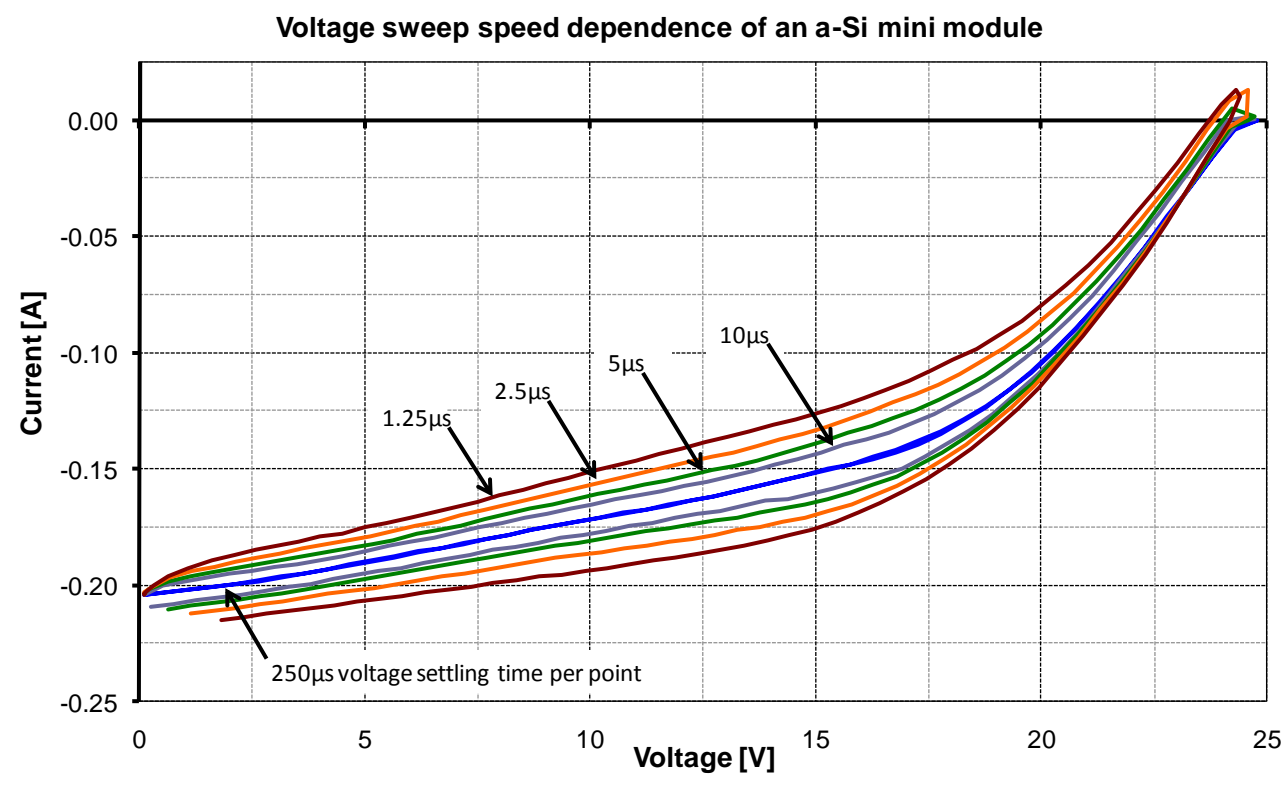

Fig. 2: Dependence of the voltage sweep rate of an a-Si module measured in both directions continuously from forward to reverse bias. The device was measured at different voltage settling times per point. An additional 1.25us per point was allowed for simultaneous voltage and current measurements. Clearly visible are increasing capacitive effects decreasing settling time for the voltage.

Furthermore, recent developments in new high power LEDs have improved the light intensity, efficiency and power output of LEDs immensely. They have the potential for reaching intensity levels of more than the $1000 \mathrm{~W} / \mathrm{m}^{2}$ required to meet STC., as demonstrated in CREST's prototype where $1.2 \mathrm{~kW} / \mathrm{m}^{2}$ is achieved. The very long lifetime of LEDs is a further advantage to all other light sources on the market. With up to 50.000 to $100.000 \mathrm{~h}$ it surpasses multiple replacements of flash or steady state sources, which not only compensates the initial higher cost per light intensity but 
also means reducing maintenance cost down to a minimum as well as largely increasing calibration accuracy over time and reducing recalibration and reclassification costs and downtime.

\subsection{Disadvantages}

One drawback of LEDs characteristics is that, even being very efficient light sources, they tend to get very hot and need rapid and efficient cooling. Due to the negative voltage temperature coefficient, the LEDs light output energy and efficiency drops with rising operation temperature of the dye. This directly leads to temporal instability during I-V measurements when the LED is current regulated (as measured in the LED-based solar simulator, see Fig. 3). The voltage temperature coefficient generally increases with wavelength, which means that red and infrared LEDs drop in light intensity with rising temperature more than blue ones. The output efficiency and intensity drop down can be overcome with a direct irradiance regulation control, which simply increases power input to overcome the light energy output change.

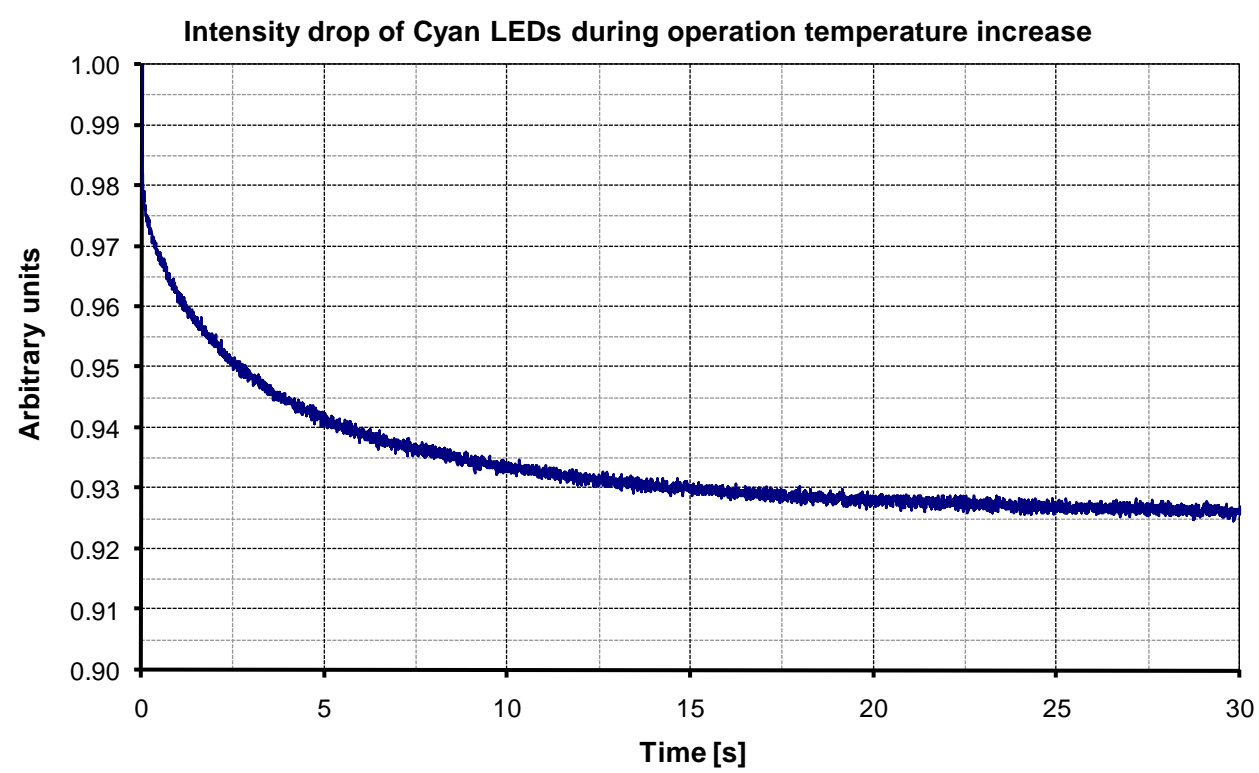

Fig. 3. Measured intensity drop down of current regulated Cyan LEDs in the LED-based solar simulator during increase of operation temperature in the first seconds after switch-on. The junction temperature at the beginning was measured at $17^{\circ} \mathrm{C}$ and the final temperature is estimated from the loss of intensity to be $60-65^{\circ} \mathrm{C}$.

The positive temperature coefficient of the dominant output wavelength or peak wavelength can also be problematic, which makes the spectral output of the LED slightly shift into higher wavelengths. Although in the LED-based simulator the wavelength shift with temperature is negligibly small (Fig. 4), good cooling of LEDs nevertheless is essential, which at the same time reduces output efficiency drop and increases lifetime.

As with virtually all kinds of light sources, LEDs also show some dependence of the spectral output spectrum to the light output (drive current). It has been observed that the spectrum slightly shifts to the lower wavelength region (Fig. 5). Although the effect is not very large, the highest accuracy during characterisation of photovoltaic devices can be achieved via monitoring not only the irradiance but also the output spectrum of the simulator throughout the measurement. In this way, changes due to temperature and drive current are monitored and known and highest accuracy in calibration can be achieved. 


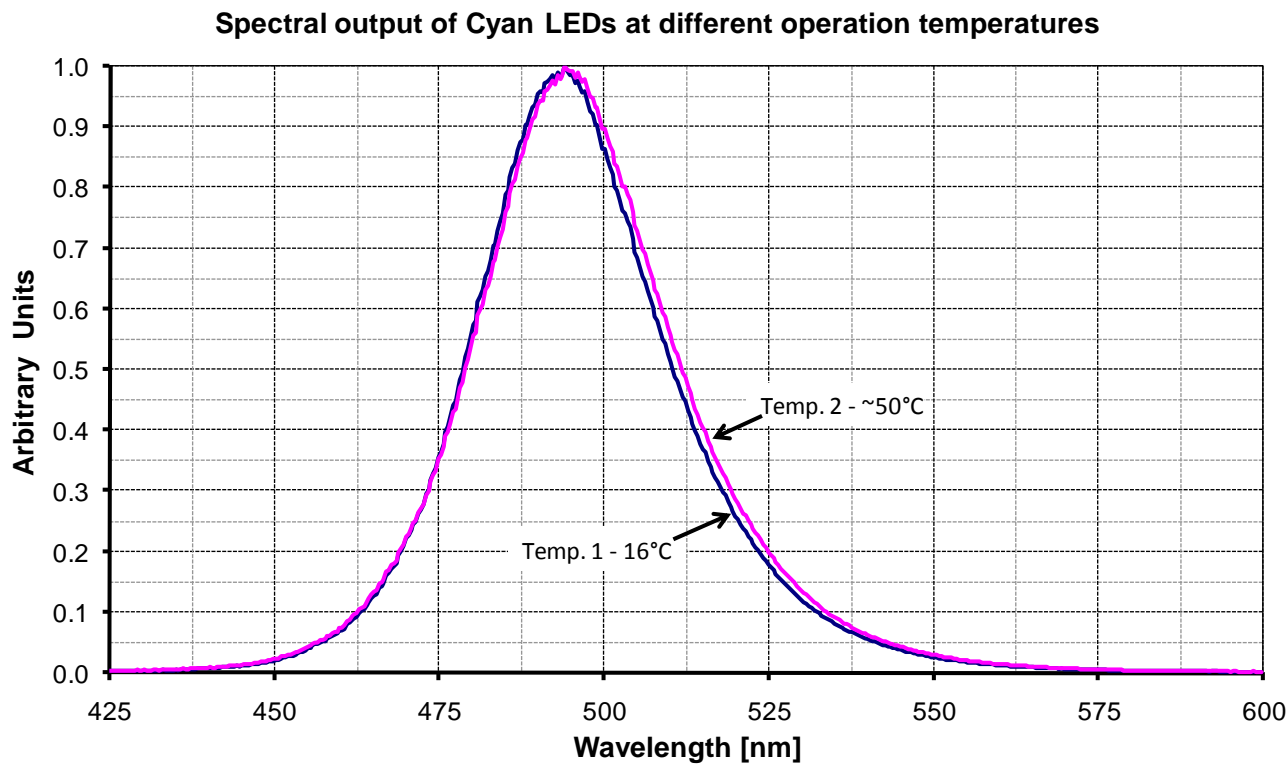

Fig. 4. Spectral output of cyan LEDs at different dye operation temperatures. A dominant wavelength shift of $1 \mathrm{~nm}$ in direction to the red region could be measured with a temperature difference of around $35^{\circ} \mathrm{C}$. It can also be observed that the spectral emission curve slightly widens as well. Nevertheless changes are minimal.

\section{Spectral changes at different intensities of Cyan LEDs}

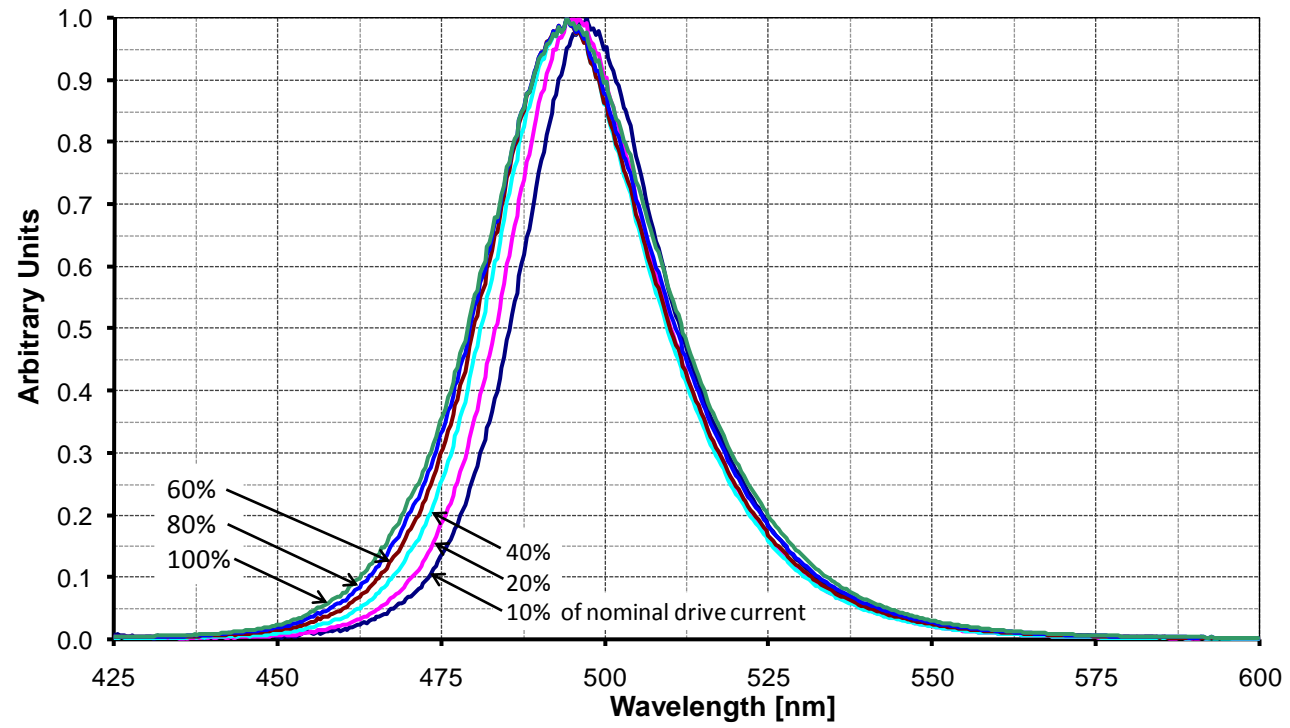

Fig. 5. Normalised spectral output of cyan LEDs at different intensities: It can be seen that the spectral output slightly increases in the blue region of the spectrum, although the LED dye temperature could not be kept stable throughout all measurements at different intensities, since they have been made directly on the LED-based solar simulator. Changes on the right side of the dominant wavelength are considered to be more influenced by operation temperature changes.

An unavoidable disadvantage is the degradation of the LEDs throughout their lifetime. Degradation-rate depends on the operation temperature of the LED and the effect can be largely reduced by appropriate cooling and by operating the LED at lower currents than rated. Since the degradation effect can only be reduced, the main solution is a system designed to initially supply higher irradiances than needed under STC, so that after degradation required conditions can still be applied. While this clearly is not desirable, such degradation is also seen with conventional light sources. 


\section{LED-BASED SOLAR SIMULATOR}

An LED-based solar simulator prototype was constructed in CREST, showing that a solar simulator can well work with LEDs at the main light sources. Different types of LED simulators have been reported in the past from h.a.l.m. electronics $^{[7]}$ and Tokyo University of Agriculture and Technology ${ }^{[8],[9]}$ but neither of these devices demonstrated the required intensities and spectrum needed for STC measurements. The presented system is the first with quasi-continuous spectrum and achieving one Sun intensity. It should be noted however, that in this proto-type development, the NIR is provided by Halogen lamps for cost reasons. It is planned to replace these with additional LEDs in the final system. In the following sections a brief description about the hardware set-up and the achieved simulator classifications can be found. The described system is a good working prototype, but not yet working perfectly and many lessons have been learned throughout analysis of the system qualities. Those possible improvements are described as well and will be implemented upon the final unit.

\subsection{Hardware set-up}

The simulator consists of 5 main parts, illustrated in a simplified hardware schematic in Fig. 7. A picture of the actual simulator with description of the location of the different parts of the system is shown in Fig. 6.

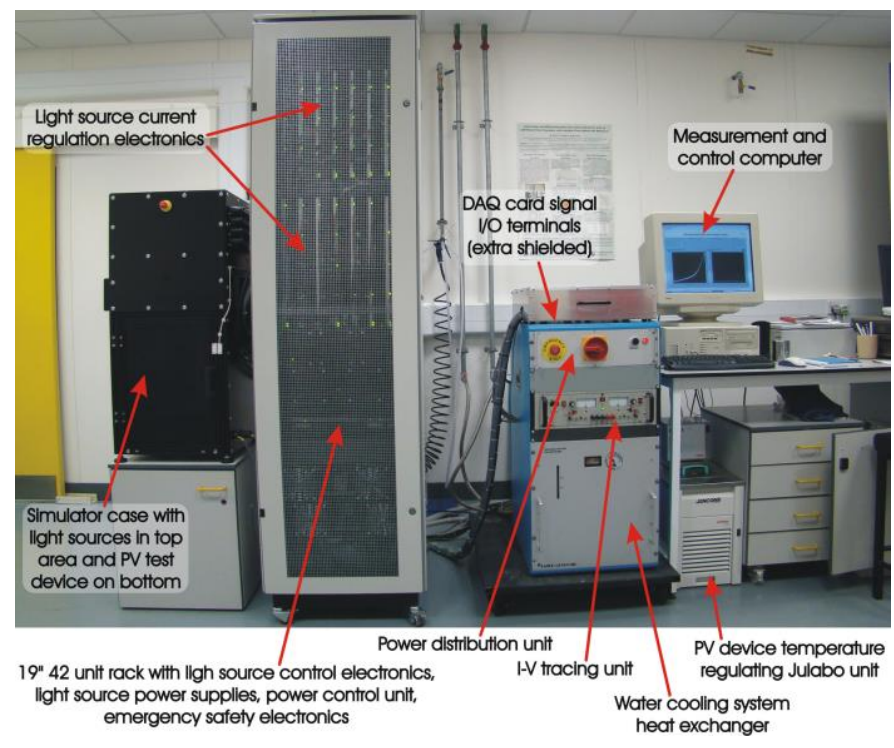

Fig. 6. LED-based solar simulator with parts description

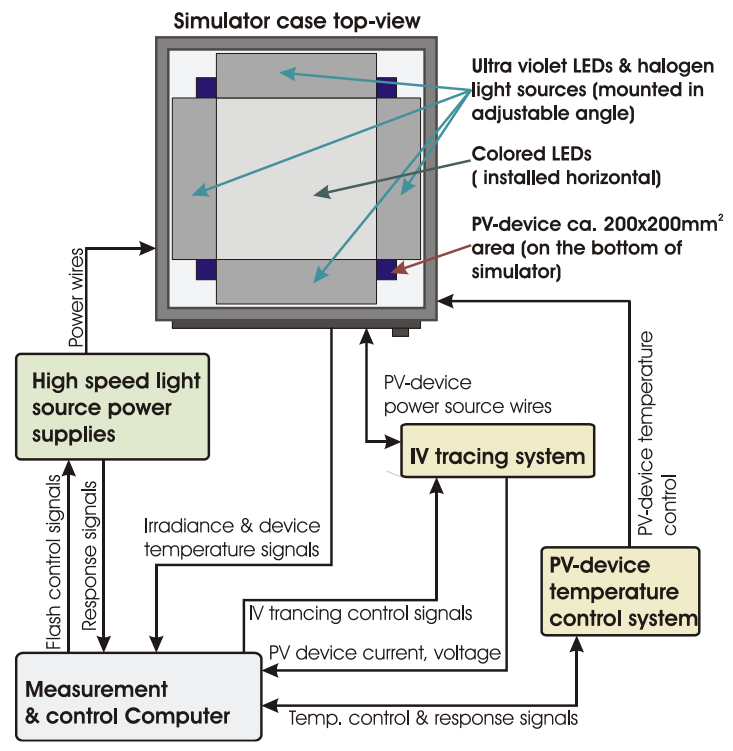

Fig. 7. Simplified simulator hardware schematic

The simulator LED array consists of several hundred LEDs in 8 different colours, to cover the light spectrum from the ultraviolet at $375 \mathrm{~nm}$ to the red at $680 \mathrm{~nm}$. In this prototype, halogen lights are used to cover the infrared part of the spectrum, while developments are ongoing to replace this with LEDs in the final product. The area of the light sources is $380 \times 380 \mathrm{~mm}$ and the distance to the illuminated test area is $650 \mathrm{~mm}$. The LEDs colours were chosen for matching the AM1.5G spectrum used in STC.

The I-V curve is traced by an analog 4-quadrant high-speed operational amplifier. Current, voltage and irradiance sensor signals are measured simultaneously, ensuring that all data directly relates to each other and no uncertainties are introduced throughout time differences between the measurement channels. Measurement speed is fully adjustable and can be as short as $2.5 \mu$ s per measurement point, including regulation time for the I-V tracer and sampling period.

A PV device temperature control system is capable of regulating the test device temperature from 10 to $80^{\circ} \mathrm{C}$, limited by the water which is used for circulation between the radiator block and the refrigerated/heating unit.

The simulator is controlled by a computer with in-house developed LabVIEW software. Measurements are completely automated after configuration. Simulator status monitoring makes sure that all parts in the complex are working fine and prevents any damage to the system in the case of an unusual event with an automated shut down, so that measurements can run for hours or days without the user having to be there all time. 


\subsection{Characterisation}

The target of the LED simulator development was to achieve a class AAA as defined in IEC60904-9 edition $2^{[3]}$. In the following, the prototype is assessed as class B, which is largely due to shortcomings of the halogen illumination rather than the LED sources. However, there are also clear avenues for improving on this which will be implemented in the device developed in the next stage of this project.

\subsubsection{Spectral output}

One big advantage in using LEDs as the main light source was mentioned to be the very good variability of the output spectrum. Hence the source spectrum (see Fig. 8) has been measured in two configurations: at full intensity of all light sources with no further intensity adjustment and optimised (by simplex fitting) with adjusted intensities for matching the AM1.5G spectrum as closely as possible.

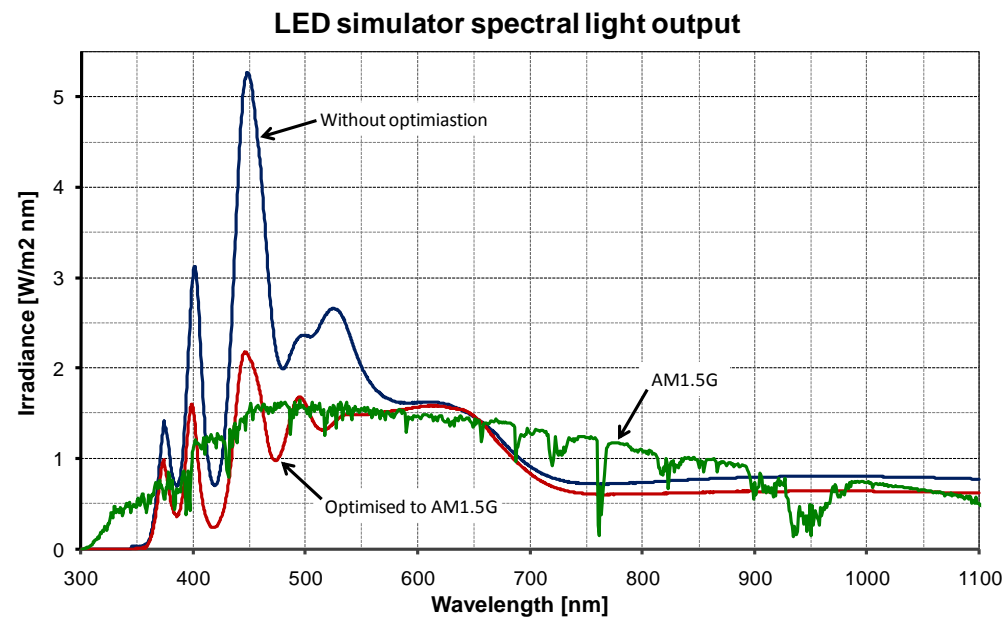

Fig. 8. Simulator spectral output measured in two configurations: at full intensity without further adjustments and fitted the AM1.5G spectrum

Table 1. Spectral match classification to the AM1.5G target spectrum with adjusted spectral output

\begin{tabular}{ccc}
\hline \hline $\begin{array}{c}\text { Wavelength } \\
\text { interval [nm] }\end{array}$ & $\begin{array}{c}\text { Relative } \\
\text { Error }\end{array}$ & Class \\
\hline \hline $400-500$ & 1.00 & $\mathrm{~A}$ \\
$500-600$ & 1.13 & $\mathrm{~A}$ \\
$600-700$ & 1.12 & $\mathrm{~A}$ \\
$700-800$ & 0.66 & $\mathrm{~B}$ \\
$800-900$ & 0.76 & $\mathrm{~A}$ \\
$900-1100$ & 1.21 & $\mathrm{~A}$ \\
Total & - & $\mathrm{B}$ \\
\hline \hline
\end{tabular}

The spectral classification according to IEC60904-9 $9^{[3]}$ is summarised in Table 1. The full intensity case results in a class $\mathrm{C}$ grading of the spectral match. Adjusting the intensity of the different light sources achieved a class B spectrum. The target of class A classification could not be achieved in the current arrangement. From Table 1, it can be seen this is due entirely to the choice of halogen lights and does not affect the possibility of an LED-only simulator achieving class A.

\subsubsection{Non-uniformity of irradiance}

A thermopile pyranometer was used to measure the light intensity over a $220 \times 220 \mathrm{~mm}^{2}$ field at $20 \times 20 \mathrm{~mm}^{2}$ resolution, as this detector is not so susceptible to spectral variations. A warm-up time period of electronics and light sources for intensity stabilization was included in every test.

As visible in Fig. 9, the non-uniformity classification of all light sources at full power over the full area of $220 \times 220 \mathrm{~mm}^{2}$ is $19.6 \%$. Class $C$ is achieved over a test area of $140 \times 140 \mathrm{~mm}^{2}$ with $8.0 \%$ inhomogeneity. On an area of $100 \times 100 \mathrm{~mm}^{2}$ we achieve a Class B with $4.0 \%$ inhomogeneity and Class A classification with $1.5 \%$ non-uniformity has been achieved in an area of $60 \times 60 \mathrm{~mm}^{2}$. 


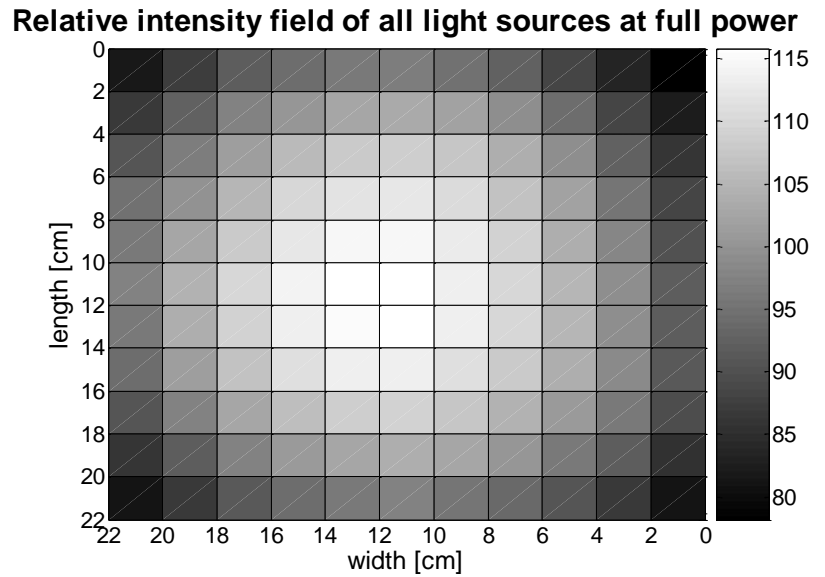

Fig. 9. Relative intensity field of all light sources at full power output

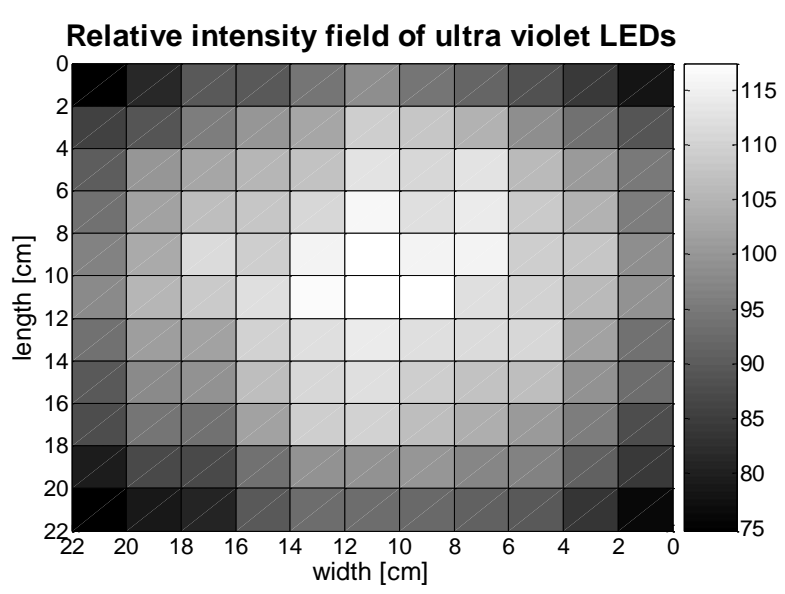

Fig. 10. Relative intensity field of all UV LEDs at full power output

Further homogeneity measurements of each individual LED colour and the halogen lights have shown that the intensity pattern changes slightly (see Fig. 10 for UV LEDs), which means that the spectral output is also changing over the illuminated area. However, due to the electronic system used in the simulator it is possible to adjust the intensity of each light source separately, which would improve the situation significantly. Work is ongoing to calculate the calibration factors to optimise the homogeneity over the illuminated area, while minimizing spectral changes.

\subsubsection{Temporal instability}

The stability of the different light sources has been measured with a silicon pyranometer Kipp \& Zonen SP-Lite, centred in the test area. This has a time constant similar to that of solar cells and thus can detect changes of relevance to solar cell calibration.

Table 2. Measured long term temporal instability and classification at different measurement times and conditions

\begin{tabular}{|c|c|c|c|}
\hline $\begin{array}{l}\text { Measurement conditions } \\
\text { and setup }\end{array}$ & Meas. time & $\begin{array}{l}\text { Long term temporal } \\
\text { instability (LTI) }\end{array}$ & Class \\
\hline All LEDs (250 $\mu$ s start-up) & $10 \mathrm{~ms}$ & $1.14 \%$ & A \\
\hline All LEDs $\left(250 \mu\right.$ s start-up $\left.{ }^{\dagger}\right)$ & $100 \mathrm{~ms}$ & $2.02 \%$ & B \\
\hline All LEDs $(250 \mu$ s start-up*) & $1 \mathrm{~s}$ & $4.03 \%$ & B \\
\hline Halogen light (2.5s start-up) & $2.5 \mathrm{~s}$ & $0.28 \%$ & A \\
\hline Halogen light (2.5s start-up) & $25 \mathrm{~s}$ & $1.49 \%$ & A \\
\hline All light sources (25s warm-up) & $24 \mathrm{~h}$ & $4.72 \%$ & B \\
\hline All light sources (15m warm-up) & $24 \mathrm{~h}$ & $0.34 \%$ & A \\
\hline
\end{tabular}

The short term instability of light intensity is specified as class $\mathrm{A}^{[3]}$, due to the simultaneous measurements of current, voltage and irradiance. Table 2 summarises the results of the long term temporal instability characterisation for different time steps. Irradiance changes during short time pulse measurements with only the LEDs switched to full power output are mainly due to the negative voltage temperature coefficients of LEDs. The intensity of the LEDs decreases until the operation temperature stabilizes, which takes approximately 40 seconds. This effect is due to the use of current regulation of the LEDs. Light intensity variation of the halogen light happens mainly the because of warm-up of electrical components in the regulation circuits. During this time the light intensity of the halogen lights drops slightly.

\footnotetext{
$\dagger$ A slightly longer start-up would reduce LTI and thus let us achieve class A
} 
The complete warm-up and stabilization process of the light intensity takes around 15 minutes. As seen in Table 2 in a $24 \mathrm{~h}$ test, the intensity changes after this period are only minor and the simulator can be classified with a class $\mathrm{A}^{[3]}$ temporal instability during a $24 \mathrm{~h}$ test duration.

\subsubsection{Light intensity}

The total irradiance has been measured with thermopile pyranometer responding linearly from $310 \mathrm{~nm}$ to $2800 \mathrm{~nm}$. At full power output of all light sources an irradiance of $1.4 \mathrm{~kW} / \mathrm{m}^{2}$ has been measured in the centre of the illumination area. Adjusting the spectrum to match AM1.5G reduces the maximum irradiance to $1.2 \mathrm{~kW} / \mathrm{m}^{2}$. The high intensity is mainly due to the halogen lights, which have a spectral output far into the infrared region with higher intensities than that of the AM1.5G spectrum. Using the spectral output data from section 3.2.1 reveals that under best fitting for the AM1.5G spectrum the total irradiance up to $1100 \mathrm{~nm}$ is only $685 \mathrm{~W} / \mathrm{m}^{2}$, which is lower than the defined $802 \mathrm{~W} / \mathrm{m}^{2}$ at $\mathrm{AM} 1.5 \mathrm{G}$ in the standard. This results in a lower irradiance than under STC when for example measuring silicon solar cells, having a response up to $1100 \mathrm{~nm}$. When using only the LEDs, an irradiance of up to $540 \mathrm{~W} / \mathrm{m}^{2}$ can be reached at full power, which means that with only LEDs the actual target of $1 \mathrm{Sun}\left(1 \mathrm{~kW} / \mathrm{m}^{2}\right.$ intensity under the full range of the sunlight spectrum) has not yet been reached.

\subsection{Possible and planned improvements}

Analysing the characterisation data gives clear indications that the LED-based solar simulator prototype has some shortcomings. Nevertheless, with its functionality and the ability to deliver a variable quasi-continuous wavelength output, it also proves that the main concept works and needs only minimal refinements.

The first thing to improve with the next incarnation is the output spectrum with intensity and homogeneity. With 8 different LED colours a good spectral match could be achieved up to a wavelength of 680nm. This will be enhanced by using many more different LED colours in the full range of $350 \mathrm{~nm}$ to $1300 \mathrm{~nm}$ (see initial simulations in Fig. 11) and thus replace the remaining halogen light sources. Newly developed high power LED sources enable us to achieve the required total light intensity of $1000 \mathrm{~W} / \mathrm{m}^{2}$. The main challenge will be achieving a class A light homogeneity over the whole test area. It is believed that an intensity calibration of every light source, as possible in the prototype, might not be enough to reach this requirement. Currently ongoing are developments of specialised optics, which first mix all incoming light and then concentrate it onto the illumination area. This could lead to a much better homogeneity of all colours throughout the field and also increase the efficiency of light collection, since a large part of light energy in the prototype actually does not hit the test area and is wasted.

\section{Simulated spectral light output in the new version}

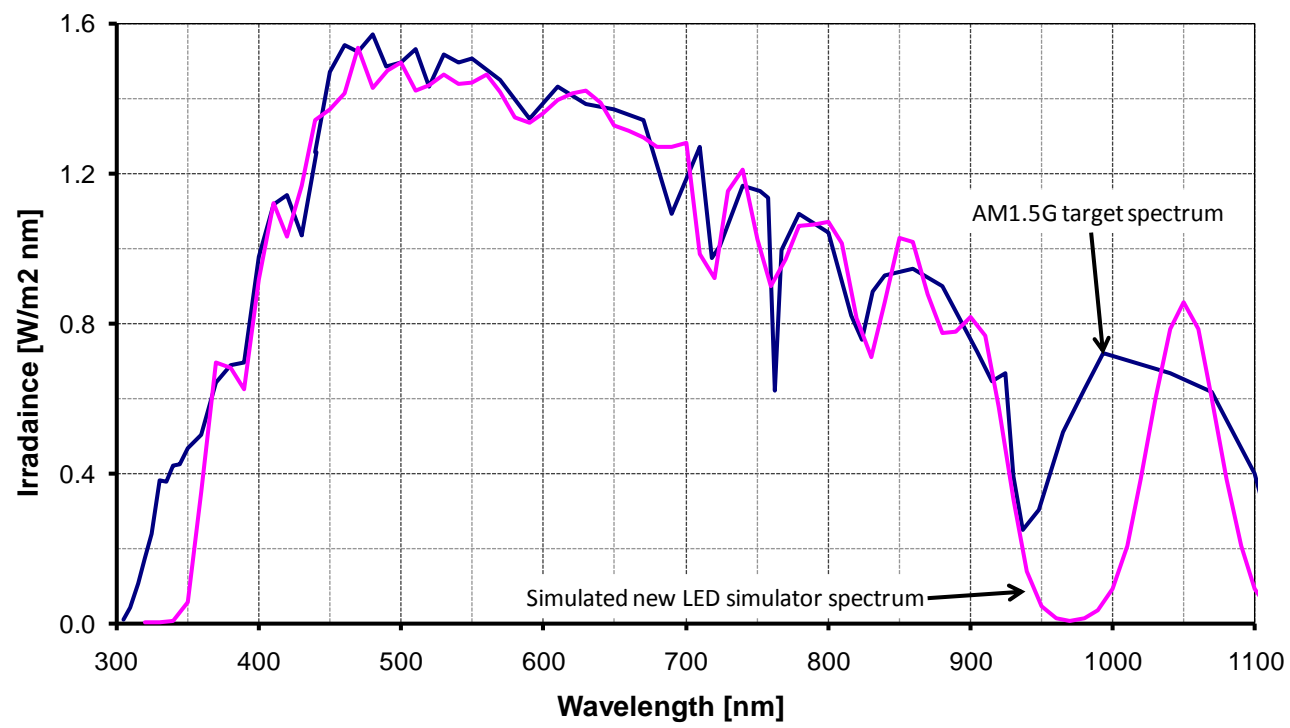

Fig. 11: Simulated spectral output of the new version of the LED-based solar simulator; development is still ongoing to improve the $950 \mathrm{~nm}$ to $1020 \mathrm{~nm}$ region. 
In terms of temporal instability of the light intensity throughout the measurement time it is planned to improve the efficiency of the cooling system to keep LEDs cooler and thus increase lifetime. Secondly, it is planned to improve the regulation electronics of the LEDs by using a cascade intensity / current regulation. As illustrated in Fig. 12, this would be a two stage regulation process with an inner drive current regulation of the LEDs and an outer intensity regulation circuit controlling the drive current from the base-LED intensity signal sensors. This kind of control increases the drive current of the light source as it heats up and the voltage drops down and thus would deliver a highly stable light output with the additional benefit of a better spectral control, since intensity and not LED drive current is the main controlling factor. The type of regulation has been used successfully in other types of solar simulators, stabilising intensity variations from power fluctuations.

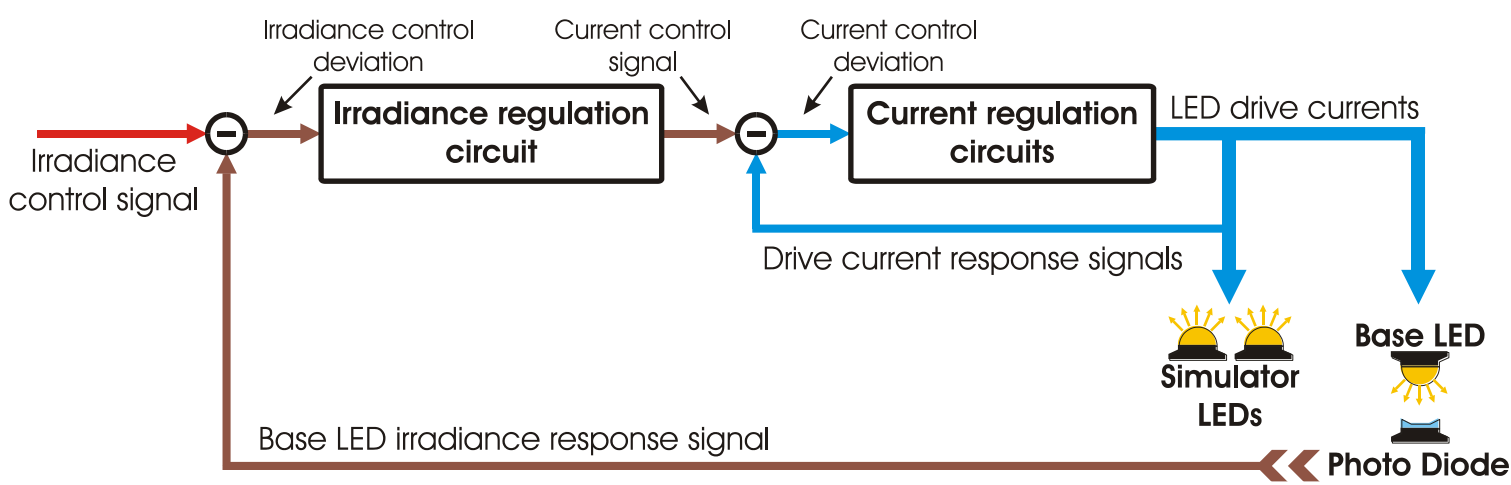

Fig. 12. Basic cascaded intensity / current regulation circuit for LEDs: The inner current regulation circuit stably drives the current of the simulator and base LEDs using their current response signals. The current control signal is provided from the outer irradiance regulation circuit, which is driven by the irradiance control signal and gets response from the base LEDs intensity signal from a photodiode. This ensures highly accurate and stable irradiance control of all LEDs per colour.

\section{CONCLUSION}

The very good characteristics of LEDs have been shown to outweigh their drawbacks and make them a virtually perfect candidate for an advanced solar simulator for measuring characteristics of all PV device types and materials. Using LEDs instantly combines flash and steady state solar simulators opening the possibility to make the most out of both system qualities. Additionally, the variety of spectrum and intensity in a full LED simulator opens possibilities for a whole new range of measurement functions and thus allows for better characterisation of all different PV device materials and structures.

The analysis of a prototype LED simulator has shown that it has the potential to deliver quality PV device measurements. Measuring solar cells within an area of $60 \times 60 \mathrm{~mm}^{2}$, the simulator has achieved a class BAA (spectral match class B, irradiance non-uniformity and temporal stability class A). The class B spectral match is entirely due to the choice of LEDs and use of halogen lights in the prototype and does not mean that achieving class A is impossible using only LEDs. Improvements mentioned show that achieving the required intensities and qualities is possible, but not trivial. The shortcomings of the prototype will be improved upon in the final unit. Furthermore, the rapid improvement of LED technology will make the overall energy delivery, spectral matching and control even better.

\section{ACKNOWLEDGEMENTS}

The design and construction of the LED-based solar simulator prototype and its new incarnation is supported by EPSRC (EP/D078431/1). 


\section{REFERENCES}

[1] Monokroussos C., Gottschalg R., Tiwari A. N. and Infield D. G., "Measurement Artefacts due to the use of Flash Simulators," PROCEEDINGS OF THE PHOTOVOLTAIC SCIENCE APPLICATION AND TECHNOLOGY CONFERENCE (PVSAT-2), 143-151 (2005).

[2] "IEC 60904-3: Measurement Principles for Terrestrial PV Solar Devices with Reference Spectral Irradiance Data," (1989).

[3] "IEC 60904-9 Ed. 2: Photovoltaic devices - Part 9: Solar simulator performance requirements," (2007).

[4] Zheludev N., "The life and times of the LED - a 100-year history," Nature Photonics 1(4), 189-192 (2007).

[5] Matson R. J., Emery K. A. and Bird R. E., "Terrestrial solar spectra, solar simulation and solar cell short-circuit current calibration: a review," Solar Cells 11, 105-45 (1984).

[6] Seifer G., Baur C., Meusel M., Dimroth F., Bett A. W. and Warta W., "Influence of the Simulator Spectrum on the Calibration of Multi-Junction Solar Cells under Concentration," CONFERENCE RECORD IEEE PHOTOVOLTAIC SPECIALISTS CONFERENCE, 836-839 (2002).

[7] Grischke R., Schmidt J., Albert H., Laux A., Metz A., Hilsenberg U. and Gentischer J., "5BV.2.47 LED Flasher Arrays (LFA) for an Improved Quality Control in Solar Cell Production Lines," EUROPEAN PHOTOVOLTAIC SOLAR ENERGY CONFERENCE, vol. 2, 2591-2594 (2004).

[8] Kohraku S. and Kurokawa K., "NEW METHODS FOR SOLAR CELLS MEASUREMENT BY LED SOLAR SIMULATOR," 1977-1980 (2003).

[9] Kohraku S. and Kurokawa K., "A fundamental experiment for discrete-wavelength LED solar simulator," Solar Energy Materials and Solar Cells 90, 3364 (2006).

[10] Baur C. and Bett A. W., "Measurement Uncertainties of the Calibration of Multi-Junction Solar Cells," CONFERENCE RECORD IEEE PHOTOVOLTAIC SPECIALISTS CONFERENCE, 583-586 (2005).

[11] Willett D., Kuriyagawa S., Institute of Electrical and Electronic Engineers and Electron Devices Society, "The Effects of Sweep Rate, Voltage Bias and Light Soaking on the Measurement of Cis-Based Solar Cell Characteristics," CONFERENCE RECORD IEEE PHOTOVOLTAIC SPECIALISTS CONFERENCE, vol. 23, 495-500 (1993). 